OPEN ACCESS

Edited by:

Elena Rybnikova,

Pavlov Institute of Physiology, Russian

Academy of Sciences, Russia

Reviewed by:

Jacques Barbet,

Arronax, France

Knut Stieger

Justus Liebig Universität Gießen,

Germany

${ }^{*}$ Correspondence:

Peijian Yue

yue_peijian@163.com

tThese authors have contributed equally to this work.

Specialty section: This article was submitted to

Neurodegeneration

a section of the journal

Frontiers in Neuroscience

Received: 09 January 2018 Accepted: 20 March 2018

Published: 06 April 2018

Citation:

Yue P, Miao W, Gao L, Zhao X and Teng J (2018) Ultrasound-Triggered Effects of the Microbubbles Coupled to GDNF Plasmid-Loaded PEGylated Liposomes in a Rat Model of Parkinson's Disease.

Front. Neurosci. 12:222. doi: 10.3389/fnins.2018.00222

\section{Ultrasound-Triggered Effects of the Microbubbles Coupled to GDNF Plasmid-Loaded PEGylated Liposomes in a Rat Model of Parkinson's Disease}

\author{
Peijian Yue ${ }^{1 * t}$, Wang Miao ${ }^{2 \dagger}$, Lin $\mathrm{Gao}^{2}$, Xinyu Zhao ${ }^{1}$ and Junfang Teng ${ }^{1}$ \\ ${ }^{1}$ Department of Neurology, The First Affiliated Hospital of Zhengzhou University, Zhengzhou, China, ${ }^{2}$ Department of \\ Neurological Intensive Care Unit, The First Affiliated Hospital of Zhengzhou University, Zhengzhou, China
}

Background: The purpose of this study was to investigate ultrasound-triggered effects of PEGylated liposomes-coupled microbubbles mediated gene transfer of glial cell line-derived neurotrophic factor (GDNF) plasmid (PLs-GDNF-MBs) on behavioral deficits and neuron loss in a rat model of Parkinson's disease (PD).

Methods: The unloaded PLs-MBs were characterized for particle size, concentration and zeta potential. PD rat model was established by a unilateral 6-hydroxydopamine (6-OHDA) lesion. Rotational, climbing pole, and suspension tests were used to evaluate behavioral deficits. The immunohistochemical staining of tyrosine hydroxylase $(\mathrm{TH})$ and dopamine transporter (DAT) was used to assess the neuron loss. The expression levels of GDNF and nuclear receptor-related factor 1 (Nurr1) were determined by western blot and QRT-PCR analysis.

Results: The particle size of PLs-MBs was gradually increased, while the concentration and absolute zeta potential were gradually decreased in a time-dependent manner after injection. 6-OHDA elevated amphetamine-induced rotations and decreased the $\mathrm{TH}$ and DAT immunoreactivity compared to sham group. However, these effects were blocked by the PLs-GDNF-MBs. In addition, the mRNA and protein expression levels of GDNF and Nurr1 were increased after PLs-GDNF-MBs treatment.

Conclusions: The delivery of PLs-GDNF-MBs into the brains using MRI-guided focused ultrasound alleviates the behavioral deficits and neuron loss in the rat model of PD.

Keywords: GDNF, microbubbles, Parkinson's disease, PEGylated liposomes, ultrasound

\section{INTRODUCTION}

Parkinson's disease (PD) is a common neurodegenerative disease, which is characterized by the loss of dopaminergic (DA) neurons in the substantia nigra (SN) (Proft et al., 2011). Patients with PD have some typical symptoms, including tremor, rigidity, and bradykinesia (Kim et al., 2011). Although multiple attempts have been made to establish therapies for PD, neither a cure nor a treatment has been proven to slow progression (AlDakheel et al., 2014). Therefore, more efforts are needed to expand the therapeutic strategies. 
Glial cell line-derived neurotrophic factor (GDNF) is considered as an essential neuroprotective ligand for midbrain DA neurons (Nitta et al., 2004). It is expressed throughout the central nervous system (CNS) during development (Nosrat et al., 1996). GDNF promotes the survival, function, and neurite growth of DA neurons both in vitro and in vivo (Kumar et al., 2015). Many studies on animal models of PD have reported beneficial effects of GDNF on DA neuron survival (Patel et al., 2013; Quintino et al., 2013). Since GDNF does not cross the blood-brain barrier (BBB), intracerebral gene delivery by injection of viral vectors has been considered as a method of administration (Tenenbaum and Humbert-Claude, 2017). However, intracerebroventricularly administered GDNF showed numerous negative side effects and no prominent clinical improvements (Nutt et al., 2003). New delivery methods for GDNF are being investigated.

Magnetic resonance imaging (MRI)-guided focused ultrasound (FUS) is a noninvasive method to induce transient $\mathrm{BBB}$ disruption by increasing the temperature or creating gas bubbles in the targeted tissues (Huang Q. et al., 2012). Microbubbles (MBs) consist of a gas core stabilized by a thin shell (Deelman et al., 2010). MBs are becoming increasingly popular tools for targeted drug delivery (Sheffield et al., 2008). Ultrasound-mediated microbubble destruction technology has become a relatively safe and promising method of gene delivery in recent years (Phillips et al., 2010). Previous studies have revealed that FUS combined with microbubbles were able to induce a targeted and reversible BBB opening in rats (Deng et al., 2012), mouse (Zhao et al., 2018), rabbits (Hynynen et al., 2001), and pigs (Huang et al., 2017). Until now, various neurotrophic factors (Samiotaki et al., 2015) and genes (Lin et al., 2016) have been delivered into targeted brain regions by FUS mediated MBs to treat neurodegenerative disease. However, the DNA-carrying capacity of MBs is limited (Zhou et al., 2015).

Polyethylene glycol (PEG)ylated liposomes (PLs) are promising and valuable drug carriers due to their many advantages including good biocompatibility, good stability, and high DNA-carrying capacity (Huang, 2008; Chen et al., 2013). However, the steric barrier of the grafted PEG moiety on the surface of the liposomes reduces interaction between the delivery system and the cell surface (Chen et al., 2011). Thus, coupling gene-loaded liposomes to the microbubble surface is adopted (Lentacker et al., 2010). This approach takes full advantage of the high DNA-carrying capacity of PLs and the ultrasound-mediated BBB disruption effect of MBs (Wang et al., 2014).

The purpose of this study was to explore ultrasound-triggered effects of the GDNF plasmid gene loaded-PEGylated liposomescoupled microbubbles (PLs-GDNF-MBs) on behavioral deficits and neuron loss in a rat model of PD.

\section{MATERIALS AND METHODS}

\section{Preparation of GDNF-Loaded Pegylated Liposomes}

PEGylated liposomes (Avanti Polar Lipids Inc., USA) containing appropriate amounts of soybean phosphatidylcholine (S100-PC), 3beta [N-( $\mathrm{N}^{\prime}, \mathrm{N}^{\prime}$-dimethylaminoethane)-carbamoyl] cholesterol (DC-cholesterol) and biotinylated 1,2-distearoyl-sn-glycero3-phosphoethanolamine-n-(carboxy[polyethylene glycol]-2,000) (Bio-PEG2000-DSPE) at a molar ratio of 90:10:5 were prepared using a thin-film hydration method (Meure et al., 2008). In brief, the lipids were dissolved in chloroform and kept overnight on a rotary evaporator (RE-52C; Shanghai Yaguang Instrument Co Ltd, Shanghai, China) at $40^{\circ} \mathrm{C}$ for solvent evaporation. After evaporation, a thin lipid film was formed and dried further in a vacuum for $8 \mathrm{~h}$ to remove the residual solvent. The lipid film was hydrated for $8 \mathrm{~h}$ using $2.4 \mathrm{ml}$ sterile phosphate-buffered solution (PBS) containing pDC315-GDNF plasmid (5 $\mu \mathrm{g}$ ) which was constructed by inserting GDNF cDNA of rat into pDC315 vector (Microbix Biosystems Inc., Toronto, Canada). The dispersion was sonicated for $5 \mathrm{~min}$ and then extruded 12 times through a polycarbonate membrane ( $100 \mathrm{~nm}$ pore size) using a miniextruder (Avanti Polar Lipids Inc., USA). The plasmid was separated from the encapsulated one by Sepharose CL-4B column chromatography.

\section{Preparation of Microbubbles}

Microbubbles were prepared according to the procedures described previously (Liu et al., 2011). Briefly, 1,2-distearoylsn-glycero-3-phosphocholine (DSPC) (5 mg), DSPE-PEG2000 (0.5 mg), Bio-PEG2000-DSPE ( $1 \mathrm{mg})$, glycerol (50 $\mu \mathrm{l})$, and PBS $(450 \mu \mathrm{l})$ were mixed in a glass container. The mixture was placed in a $60^{\circ} \mathrm{C}$ water bath for $30 \mathrm{~min}$. The lipid suspension was subsequently lyophilized. The dried powder was dispersed in $1 \mathrm{ml}$ of a liquid mixture of $50 \%$ glucose, propylene glycol, and glycerin in a volume ratio of $8: 1: 1$. Then the mixture was sonicated for $30 \mathrm{~s}$ in the presence of perfluoropropane $\left(\mathrm{C}_{3} \mathrm{~F}_{8}\right)$ gas. Subsequently, it was mechanically vibrated at $4 \mathrm{kHz}$ for $50 \mathrm{~s}$ in a dental amalgamator (YJT Medical Apparatuses and Instruments, Shanghai, China). The generated biotinylated microbubbles were washed and resuspended in PBS. Microbubbles were avidinylated by incubating with avidin $(0.025 \mathrm{mg}$ of avidin per $\mathrm{ml}$ of microbubbles; Cell Sciences, Canton, USA) for $5 \mathrm{~min}$.

\section{Coupling of Biotinylated Liposomes to Avidinylated Microbubbles}

The biotinylated liposomes (one vial) were mixed with avidinylated microbubbles $(2 \mathrm{ml})$ and incubated at room temperature for $10 \mathrm{~min}$. The liposome-coupled microbubbles were washed with PBS and stored at $-20^{\circ} \mathrm{C}$ for further use.

\section{Characterization of PEGylated Liposomes-Microbubbles (PLs-MBs) Complexes}

The particle size and zeta potential of PLs-MBs complexes were determined according to previous reports (Matos et al., 2004). The PLs-MBs complexes $(100 \mu \mathrm{l})$ were diluted with deionized water $(900 \mu \mathrm{l})$ and were measured by the dynamic light scattering using a zeta potential/particle sizer Nicomp 380 ZLS (Particle Sizing Systems, Santa Barbara, CA).

The concentration of PLs-MBs complexes was measured by ultraviolet absorbance (Dewitte et al., 2015). Briefly, $2 \mathrm{ml}$ 
of the PLs-MBs complexes was dissolved in $8 \mathrm{ml}$ ethanol. After vortexing for $10 \mathrm{~min}$, this suspension was filtered with polycarbonate membrane filters with $0.22 \mu \mathrm{m}$ pores to remove undissolved materials. The ultraviolet absorbance of each sample was then measured at $458 \mathrm{~nm}$ (UV-2401PC, Shimadzu, Japan).

\section{Animals}

Forty healthy Sprague-Dawley rats (10 rats per group; $200-$ $250 \mathrm{~g}$ ), half male and half female, were used in this experiment. Rats were housed in a temperature- and humidity controlled environment under specific pathogen-free conditions. Rats were given access to water and food ad libitum. The experimental design was approved by the Ethics Committee of The First Affiliated Hospital of Zhengzhou University. All animal experimental procedures were approved according to the guidelines of the Care and Use of Laboratory Animals by the National Institute of Health, China.

\section{6-OHDA Lesion Surgery}

The rats received a unilateral 6-OHDA lesion of the right substantia nigra (SN) (Metz and Whishaw, 2002). The rats were anesthetized by intraperitoneal injection of $10 \%$ chloral hydrate. After that, the rats were placed in a stereotaxic apparatus (Stoelting Instruments, Wood Dale, IL; USA). Then 6-OHDA [ $5 \mu \mathrm{g} / \mathrm{per}$ rat in $2 \mu \mathrm{l}$ saline with $0.2 \%$ (w/v) ascorbic acid] were injected into the $\mathrm{SN}$ of rats at the following stereotaxic coordinates: $5.2 \mathrm{~mm}$ posterior to bregma, $1.0 \mathrm{~mm}$ lateral to the midline, and $8.5 \mathrm{~mm}$ ventral to the skull surface. Then 6-OHDA was infused by infusion pump at the flow rate of $1 \mu \mathrm{l} / \mathrm{min}$. Shamoperated rats received the same procedure except $2 \mu \mathrm{l}$ vehicle of 6 -OHDA ( $0.9 \%$ saline containing $0.2 \%$ ascorbic acid) was infused into the SN. After surgery, the skin was sutured and cleaned, and the rats were allowed to recover on a heating pad before they were returned to their home cage. After 1 week, the PLs-MBs were given to rats.

\section{MRI-Guided Focused Ultrasound (FUS)}

Thirty rats with PD prepared as described above were randomly divided into three groups $(n=10)$ as follows: PBS, PLsMBs+FUS, and PLs-GDNF-MBs+FUS. Apart from the PBS group, the rats were all undergone MRI-guided-FUS.

Animals were anesthetized and placed supine on a positioning sled for MRI. Brain ultrasound treatment was conducted under the following settings: $1 \mathrm{MHz}, 20 \%$ duty cycle, and an ultrasound intensity of $2 \mathrm{~W} / \mathrm{cm}^{2}$ (Wang et al., 2014). After injecting PLsMBs $(0.01 \mathrm{ml} / \mathrm{kg}$ body weight $)$ and PLs-GDNF-MBs $(0.01 \mathrm{ml} / \mathrm{kg}$ body weight) into the tail vein for $30 \mathrm{~min}$, FUS was performed. The brain of each rat was sonicated from the dorsal surface into the right hemisphere at a depth of $2-3 \mathrm{~mm}$. After the sonication procedure, Evans blue (EB) (100 mg/kg; Sigma, USA) was injected through the tail vein to confirm the site of $\mathrm{BBB}$ disruption on tissue blocks.

Animals were intravenously injection of the PBS, PLs-MBs, and PLs-GDNF-MBs once every 3 days. The rats were sacrificed at 3 weeks after treatment.

\section{Behavior Tests}

\section{Rotational Behavior Test}

Rotational behavior was performed as previously described (Pranski et al., 2013). Animals received $2.5 \mathrm{mg} / \mathrm{kg}$ D-amphetamine intraperitoneally. One hour after injection, rats were monitored for rotation behavior for $30 \mathrm{~min}$. Rotations ipsilateral to the lesion were counted as a positive value, while rotations toward the contralateral side of the lesion were counted as a negative value.

\section{Suspension Experiment}

The test was performed as previously reported (Meng et al., 2017). Briefly, the rats were placed on a horizontal wire of $\sim 1.5 \mathrm{~mm}$ in diameter, suspended $30 \mathrm{~cm}$ from the ground. The hang time was recorded to detect rat limb coordination. Scoring criteria: $0-5 \mathrm{~s}, 0$ points; $6-10 \mathrm{~s} 2$ points; $11-15 \mathrm{sec}, 3$ points; $16-20 \mathrm{~s}, 4$ points; and $>20 \mathrm{~s}, 5$ points. The mean values were calculated.

Climbing pole test: The climbing pole test was conducted as previously described (Liu et al., 2016). Briefly, a rat was placed at the top of a pole with a length of 60 and $1.0 \mathrm{~cm}$ in diameter. The times for climbing down the upper half of the pole, for climbing down the lower half of the pole and for climbing down the total length of the pole were recorded. And the results were scored as 3 , within $3 s ; 2$, within $6 s$ and 1 , over $6 s$. The results were expressed as the total score.

\section{Immunohistochemistry}

Animals were sacrificed via pentobarbital overdose (60 $\mathrm{mg} / \mathrm{kg}$ ) and intracardially perfused with saline, then $4 \%$ paraformaldehyde. Brains were removed and post-fixed in $4 \%$ paraformaldehyde for $24 \mathrm{~h}$ and then dehydrated in $30 \%$ sucrose. The sections at a thickness of $16 \mu \mathrm{m}$ were cut through the substantia nigra $(-2.92$ to $-3.88 \mathrm{~mm}$ from bregma) (Paxinos and Watson, 2007). The tissue sections were incubated in $0.3 \% \mathrm{H}_{2} \mathrm{O}_{2}$ for $45 \mathrm{~min}$, and blocked in $10 \%$ goat serum for $1 \mathrm{~h}$. The sections were incubated overnight at $4^{\circ} \mathrm{C}$ with rabbit anti-tyrosine hydroxylase (TH; 1:300; Millipore), rabbit anti-dopamine transporter (DAT; 1:200; Santa Cruz), rabbit anti-GDNF (1:200; Santa Cruz Biotechnology) or rabbit antiNurr1 (1:100; Santa Cruz Biotechnology). Subsequently, the sections were incubated with biotinylated goat anti-rabbit IgG antibody (1:250; Vector labs) and avidin-biotin complex (ABC-kit, Vector Labs) at $37^{\circ} \mathrm{C}$ for $30 \mathrm{~min}$, and then stained with 3,3'-diaminobenzidine (DAB). Then the sections were observed under a light microscope (BX51; Olympus, Tokyo, Japan). Image scanning analysis system (Image-Pro Plus) was used to analyze the changes in integrated optical density (IOD) of DAT, TH and GFAP.

\section{Quantitative Real-Time PCR (qRT-PCR)}

Total RNA was isolated using Trizol (Invitrogen, Carlsbad, CA) from the frozen brains of rats. cDNA synthesis was performed using a High Capacity cDNA Reverse Transcription Kit (Applied Biosystems, USA) according to the manufacturer's instructions. QRT-PCR was performed by using SYBR green master PCR mix (Applied Biosystems, USA) on a 7,500 real-time PCR 
system (Applied Biosystems). The glyceraldehyde-3-phosphate $(\mathrm{GAPDH})$ was used as an internal control. Primers used for analyses were listed below.

\section{Gdnf-F: 5' -CGGACGGGACTCTAAGATGA-3'}

Gdnf-R: 5' -CGTCATCAAACTGGTCAGGA-3'

Nurr1-F: 5'-CAACTACAGCACAGGCTACGA-3'

Nurr1-R: 5'-GCATCTGAATGTCTTCTACCTTAATG-3'

Gapdh-F: 5'-GTGAAGGTCGGTGTCAACGGATTT-3'

Gapdh-R: 5'-CACAGTCTTCTGAGTGGCAGTGAT-3'

\section{Western Blot Analysis}

Tissue samples were lysed in RIPA buffer in the presence of protease inhibitors (Roche, USA). Equal amounts of proteins $(50 \mu \mathrm{g})$ were separated on $10 \%$ SDS-polyacrilamide gel and then transferred to PVDF membranes (Amersham). After being blocked in $5 \%$ non-fat milk for $1 \mathrm{~h}$, the membrane

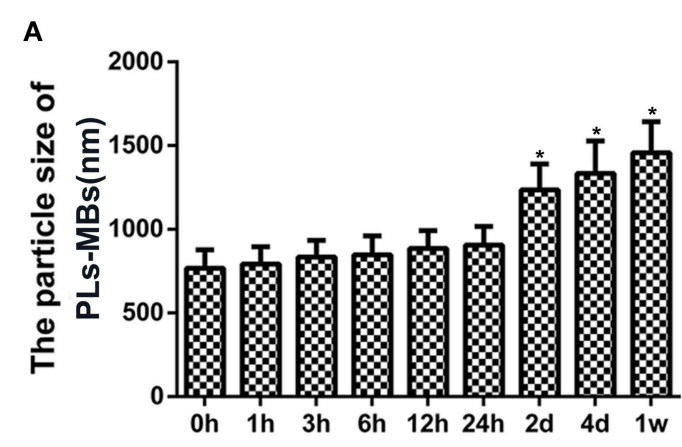

B

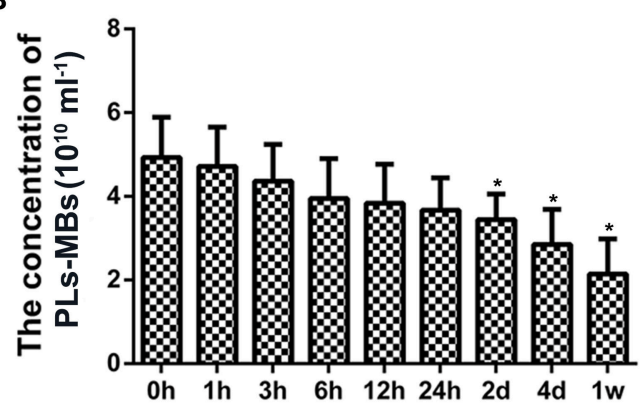

C

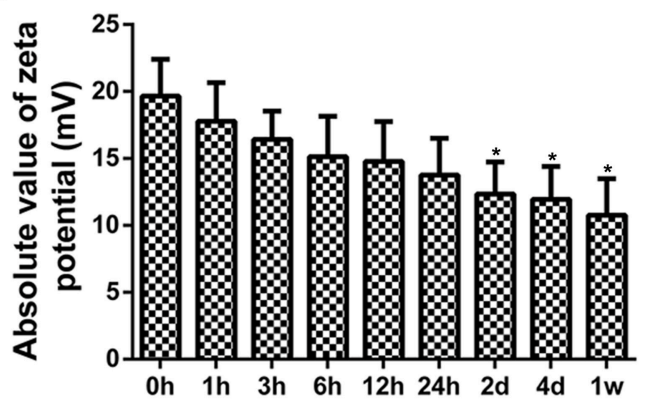

FIGURE 1 | Characterization of unloaded PLs-MBs. (A) Size, (B) Concentration, and (C) zeta potential of unloaded PLs-MBs for different duration. ${ }^{*} P<0.05$ vs. 0 h group. was incubated overnight at $4^{\circ} \mathrm{C}$ with the following primary antibodies: anti-GDNF (1:1,000; R\&D Systems), anti-Nurr1 (1:1000; Santa Cruz, USA), and anti-GAPDH (1:500; Santa Cruz, USA). After washing with TBST, the membranes were incubated with peroxidase-conjugated secondary antibody (1:10,000, Cell Signaling Technologies) for $1 \mathrm{~h}$ at $37^{\circ} \mathrm{C}$. The immunoreaction was visualized with enhanced chemiluminescence plus reagents (Millipore, USA). GAPDH was used as an internal control.

\section{Statistical Analysis}

All data are presented as means $\pm \mathrm{SD}$. All statistical analyses were performed using GraphPad Prism 6.0 software (GraphPad Prism Inc., USA). Comparison between two groups was performed by unpaired Student's $t$-test. Comparison among multiple groups was performed by one-way analysis of variance (ANOVA) with LSD post hoc test. $P<0.05$ was considered statistically significant.

\section{A}

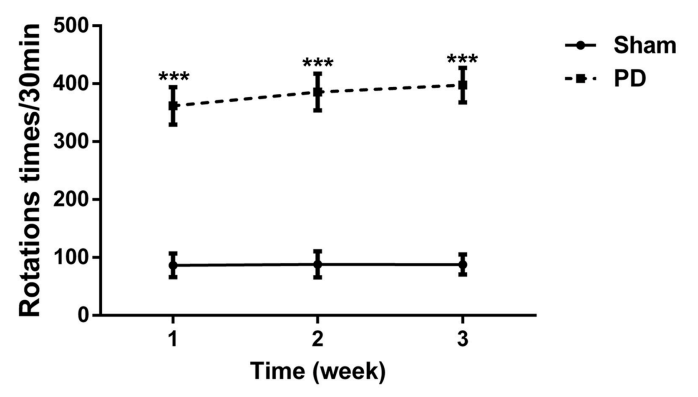

B

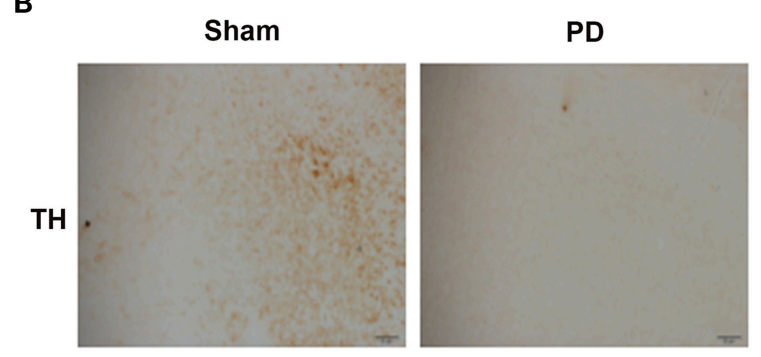

C

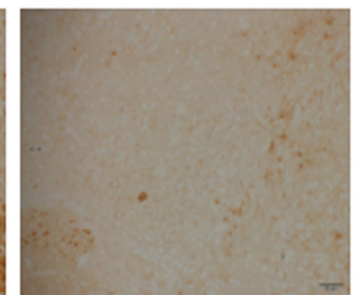

FIGURE 2 | The behavioral deficits and neuron loss in rats after unilateral injections of 6-OHDA into their SN. (A) The asymmetric rotational behavior induced by amphetamine after 6-OHDA injection for 1, 2, and 3 weeks. (B) Representative immunostainings of TH protein in the SN after 6-OHDA injection for 3 weeks. Scale bars $=50 \mu \mathrm{m}$. (C) Representative immunostainings of DAT protein in the SN after 6-OHDA injection for 3 weeks. Scale bars $=50 \mu \mathrm{m} .{ }^{\star \star \star} P<0.001$ vs. Sham group. 


\section{RESULTS}

\section{The Properties of Unloaded PLs-MBs}

Particle size, concentration, and zeta potential were measured for analysis of the properties of unloaded PLs-MBs. At $0 \mathrm{~h}$, the mean particle size of PLs-MBs was $768.24 \pm 108 \mathrm{~nm}$ with a mean concentration of $4.93 \pm 0.96 \times 10^{10} / \mathrm{ml}$, and its mean absolute value of zeta potential was $19.66 \mathrm{mV} \pm 2.75 \mathrm{~mA}$ (Figures 1A-C). With the extension of time, the particle size was gradually increased, while the concentration and absolute zeta potential were gradually decreased, suggesting the stability of PLs-MBs is gradually decreased as the time prolongs. The differences in the particle size, concentration and zeta potential became significant at day 2, the mean particle size of PLs-MBs was $1235.62 \pm 154 \mathrm{~nm}$ with a mean concentration of $3.44 \pm 0.62 \times 10^{10} / \mathrm{ml}$, and its mean absolute value of zeta potential was $12.35 \mathrm{mV} \pm 2.38 \mathrm{~mA}$ (Figures 1A-C). The ever-increasing size and decreasing zeta potential could inhibit the effect of PLs-MBs, which needs to be improved.

\section{The Behavioral Deficits and Neuron Loss in a Rat Model of PD}

To assess the effects of 6-OHDA treatment on behavioral deficits, rotational behavior test was performed. As shown in Figure 2A, the number of rotations gradually increased in the $\mathrm{PD}$ group in a time-dependent manner when compared to sham group.

To clarify the effect of 6-OHDA on DA neuron loss, immunohistochemical staining for $\mathrm{TH}$ and DAT proteins were performed. Three weeks after surgery, 6-OHDA resulted in an obvious decrease in the $\mathrm{TH}$ and DAT protein expression levels in comparison with sham group (Figures 2B,C), indicating that the PD rat model was established successfully.

\section{PLs-GDNF-MBs Alleviated the Behavioral Deficits and Neuron Loss}

To evaluate the effects of PLs-GDNF-MBs on 6-OHDAinduced behavioral deficits, PLs-GDNF-MBs were injected intravenously and delivered to the 6-OHDA lesioned striatum with MRI-guided FUS one week after unilateral partial lesioning with 6-OHDA and then the rats received three behavioral tests at 1,2, and 3 weeks following PLs-GDNF$\mathrm{MBs}$ injection. In the three behavioral experiments, there were no significant differences between PBS and PLs-MBs groups (Figures 3A-C). In Figure 3A, the apomorphine-induced rotational behavior was significantly reduced after PLs-GDNFMBs treatment as compared to the PLs-MBs group. Besides, PLsGDNF-MBs significantly up-regulated the scores in suspension and climbing pole tests when compared to PLs-MBs group (Figures 3B,C).

Subsequently, we detected the effects of PLs-GDNF-MBs on 6-OHDA-induced neuron loss. Treatment with PLs-MBs had no effect on TH and DAT immunoreactivity in the SN compared with PBS group (Figures 3D,E). By contrast, higher $\mathrm{TH}$ and DAT immunostaining in the SN of the rats injected with PLsGDNF-MBs compared to the animals treated with PLs-MBs, suggesting the PLs-GDNF-MBs attenuated the 6-OHDA-induced neurotoxicity.

\section{PLs-GDNF-MBs Increased the Expression of GDNF and Nurr1}

Next, we investigated the effects of PLs-GDNF-MBs on the expression of GDNF and Nurr1 in vivo. The results showed that the mRNA and protein expression levels of GDNF were obviously increased after PLs-GDNF-MBs treatment. In addition, the Nurr 1 mRNA and protein levels also increased after PLs-GDNFMBs treatment in vivo (Figures 4A,B). Furthermore, higher

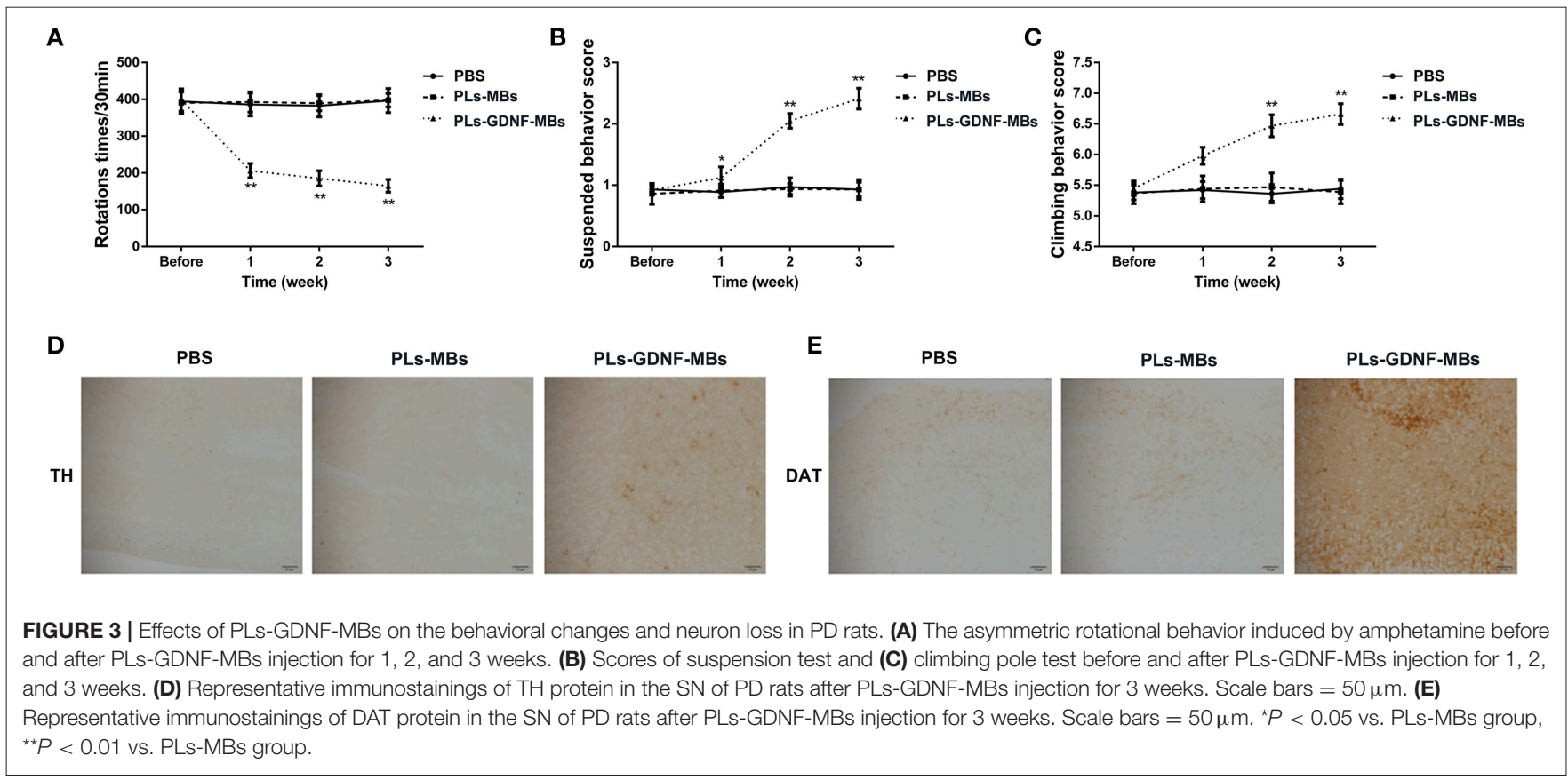


A

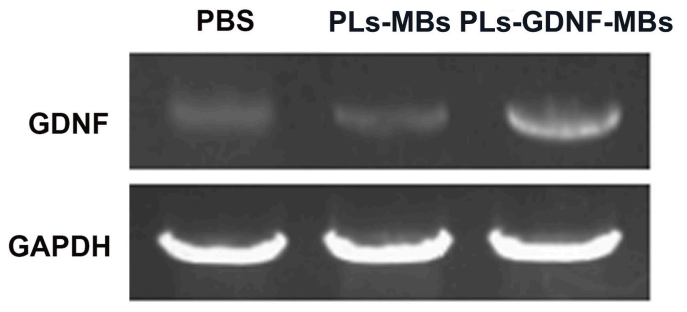

B

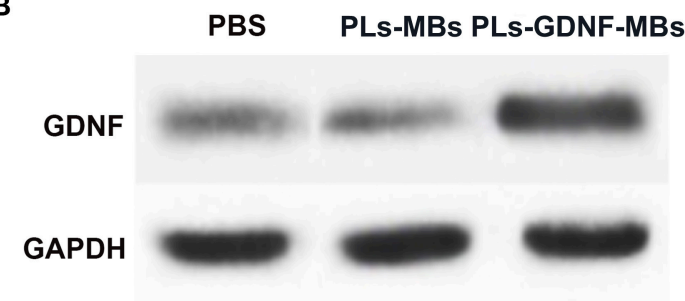

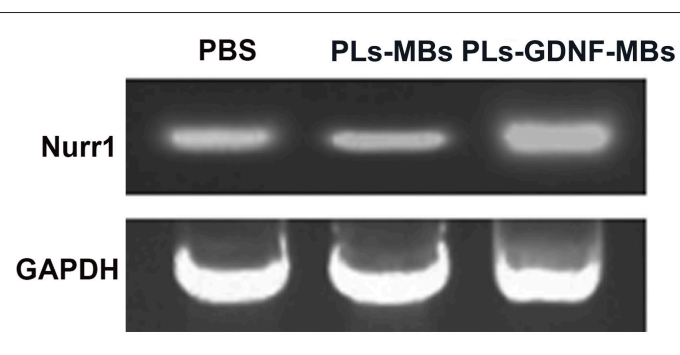

PBS PLs-MBs PLs-GDNF-MBs

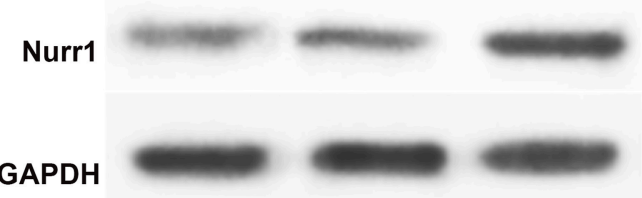

C

\section{PBS}
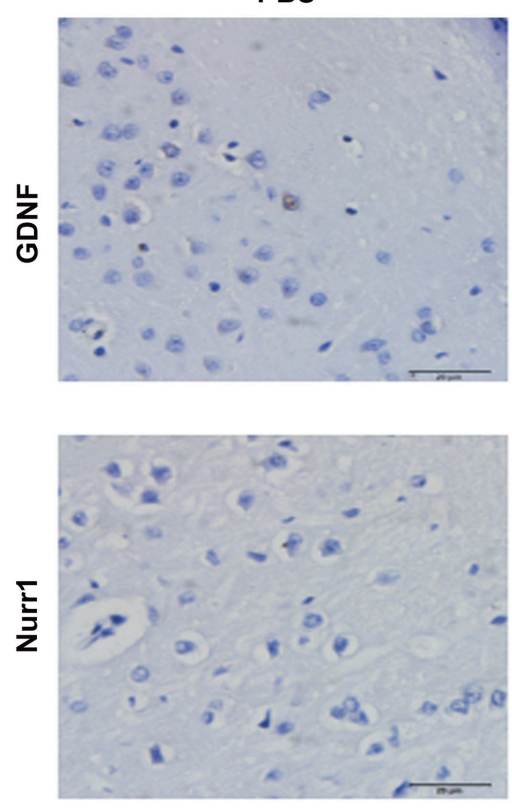
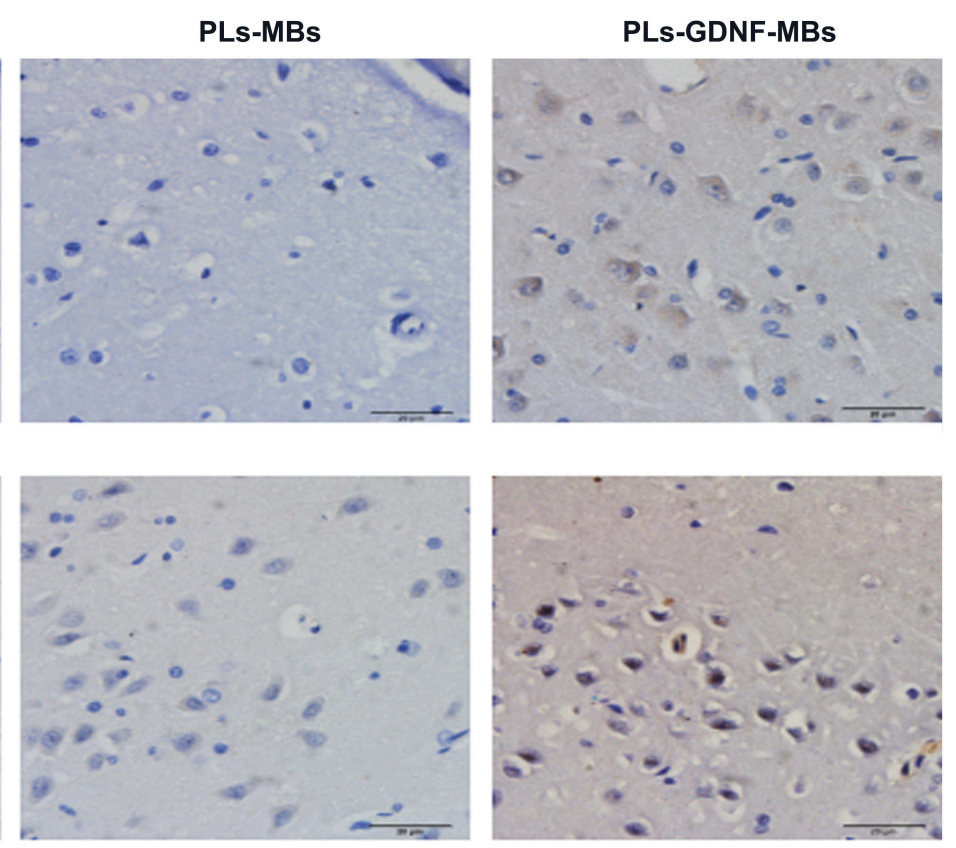

FIGURE 4 | Effects of PLs-GDNF-MBs on the mRNA and protein expression levels of GDNF and Nurr1 in PD rats. (A) QPCR analysis of GDNF and Nurr1 mRNA levels in the brain tissues of PD rats after PLs-GDNF-MBs injection for 3 weeks. (B) Western blot analysis of GDNF and Nurr1 protein levels in the brain tissues of PD rats after PLs-GDNF-MBs injection for 3 weeks. (C) Representative immunostainings of GDNF and Nurr1 proteins in the SN of PD rats after PLs-GDNF-MBs injection for 3 weeks. Scale bars $=20 \mu \mathrm{m}$.

GDNF and Nurr1 immunostaining was observed in the PLsGDNF-MBs group compared to the PLs-MBs group (Figure 4C). Taken together, these results suggest that Ultrasound-triggered PLs-GDNF-MBs promote both GDNF and Nurr1 expression in the protection against DA neuron loss.

\section{DISCUSSION}

Clinical trials of neurotrophic factor therapy for the treatment of Parkinson's disease through direct delivery of recombinant
GDNF have failed to display clinical benefit (Lang et al., 2006). Moreover, great efforts have been made for the development of in vivo gene transfer by vectors expressing the GDNF gene (Kirik et al., 2000, 2004). However, the therapeutic application of the GDNF protein or gene in the CNS is related to a major problem: the CNS is protected by the $\mathrm{BBB}$, so it is easily blocked (Wang et al., 2012).

Biochemical modifications of the nanocarriers and $\mathrm{BBB}$ disruption with FUS and MBs are promising approaches which enhance transport or bypass the BBB (Papademetriou and 
Porter, 2015). Recently, FUS combined with a specially designed plasmid-conjugated MBs have already been demonstrated the possibility of local CNS gene expression (Huang Q. et al., 2012). Hsu et al. demonstrated that the transduction of astrocytes by AAV2-GFP delivered can successfully penetrate the BBB-opened brain regions to express GFP through FUS-BBB opening (Hsu et al., 2013). Weber-Adrian et al. found that a viral vector (scAAV9-GFP) was injected intravenously and MRI-guided FUS was used to target one side of the cervical spinal cord in adult rats (Weber-Adrian et al., 2015). In our studies, as the DNA-carrying capacity of MBs is limited (Zhou et al., 2015), the PLs-MBs complexes combined with MRI-guided FUS for gene delivery were established.

It is known that reducing the particle size of delivery systems may enhance the cellular uptake of delivery systems (Kumari and Yadav, 2011). Besides, the stability of nanoparticle is very important for their practical applications, and it is closely related to its' electrokinetic properties (Huang Y. et al., 2012). Generally, nanoparticles with high absolute zeta potential are electrically stabilized and have good stability (Xu et al., 2012). Our results showed that the particle size of the PLs-MBs complexes was gradually increased, while the concentration and absolute zeta potential were gradually decreased in a time-dependent manner, suggesting the stability of PLs-MBs complexes is gradually decreased as the time prolongs. This is the deficiency of the PLs-MBs complexes, which needs further improvement.

Next, we assessed the effects of the PLs-GDNF-MBs on behavioral deficits. The apomorphine-induced rotational test was used to examine the injury degree of dopaminergic system (Tai et al., 2013). Our data showed that the number of apomorphineinduced rotations significantly increased in the PD group compared with the sham group. In contrast, PLs-GDNF-MBs treatment showed an obvious reduction in this behavioral deficit. The other two behavioral deficits were also significantly alleviated by PLs-GDNF-MBs treatment measured by climbing pole test and suspension test. These results suggest the protective effects of PLs-GDNF-MBs on attenuating behavioral deficits in the PD rat model. GDNF supports the growth and survival of dopaminergic neurons and is a potent agent for PD therapy due to its neuroprotective and neurotrophic effects (Lin et al., 1993). Previous studies have shown that GDNF plasmid gene delivery is feasible when using recombinant AAV vectors as a gene vector (Kells et al., 2012; Redmond et al., 2013).

$\mathrm{TH}$, the rate-limiting enzyme in DA synthesis, is a wellestablished marker for identification of DA neurons (Apuschkin

\section{REFERENCES}

Afonso-Oramas, D., Cruz-Muros, I., Barroso-Chinea, P., Álvarez de la Rosa, D., Castro-Hernández, J., Salas-Hernández, J., et al. (2010). The dopamine transporter is differentially regulated after dopaminergic lesion. Neurobiol. Dis. 40, 518-530. doi: 10.1016/j.nbd.2010.07.012

AlDakheel, A., Kalia, L. V., and Lang, A. E. (2014). Pathogenesis-targeted, disease-modifying therapies in Parkinson disease. Neurotherapeutics 11, 6-23. doi: 10.1007/s13311-013-0218-1

Apuschkin, M., Stilling, S., Rahbek-Clemmensen, T., Sørensen, G., Fortin, G., Herborg Hansen, F., et al. (2015). A novel dopamine transporter transgenic et al., 2015). DAT is essential for maintaining DA homeostasis and is a marker for the evaluation of the integrity of the DA system (Afonso-Oramas et al., 2010). In our study, we combined the expression of DA markers ( $\mathrm{TH}$ and DAT) to evaluate the effects of PLs-GDNF-MBs on neuron loss. Our immunohistochemical analysis showed that TH and DAT immunostaining were lower in the PD group as compared with the sham group. However, TH and DAT immunoreactivity were higher in the animals receiving PLs-GDNF-MBs for 3 weeks, indicating the neuroprotective effects of PLs-GDNF-MBs in the rats with $\mathrm{PD}$.

It has been demonstrated that GDNF promotes the expression of Nurr1 in midbrain-derived neural stem cells (Lei et al., 2011). Moreover, preclinical studies have demonstrated the essential role of Nurr1 in the midbrain DA neurons development and functional maintenance (Kadkhodaei et al., 2013). Our results showed that an increase in GDNF and Nurr1 expression levels in the PLs-GDNF-MBs group compared to the PLs-MBs group. Therefore, PLs-GDNF-MBs might relieve the neuron loss by increasing the expression of GDNF and Nurr1.

There are some limitations of this study: (1) One control appears to be missing though: what would be the effect of the infusion of GDNF-loaded microbubbles without ultrasound treatment? (2) Immunogenicity and toxicity studies should be performed because of PLs-MBs are immunogenic. (3) 6OHDA-induced PD rats did not recapitulate all the pathologic abnormalities in PD clinical cases; (4) only one-time FUS exposure was used. Repeated FUS treatment might further enhance the effect of PLs-GDNF-MBs.

In conclusion, PLs-GDNF-MBs combined with MRI-guided focused ultrasound may be an effective way of delivering the GDNF gene directly into the brain. The method provides a potential strategy of treating patients with PD.

\section{AUTHOR CONTRIBUTIONS}

PY and WM: designed the project; LG: performed the experiments; XZ: analyzed the data; JT: drafted the manuscript.

\section{ACKNOWLEDGMENTS}

This work was supported by The First Affiliated Hospital of Zhengzhou University and National Natural Science Foundation of China (81501109, U1504813) and The Education Science and Technology Projects of Henan Province, China (No.17A320067).

mouse line for identification and purification of midbrain dopaminergic neurons reveals midbrain heterogeneity. Eur. J. Neurosci. 42, 2438-2454. doi: 10.1111/ejn.13046

Chen, C. W., Lu, D. W., Yeh, M. K., Shiau, C. Y., and Chiang, C. H. (2011). Novel RGD-lipid conjugate-modified liposomes for enhancing siRNA delivery in human retinal pigment epithelial cells. Int. J. Nanomedicine 6, 2567-2580. doi: 10.2147/IJN.S24447

Chen, Y., Sun, J., Lu, Y., Tao, C., Huang, J., Zhang, H., et al. (2013). Complexes containing cationic and anionic $\mathrm{pH}$-sensitive liposomes: comparative study of factors influencing plasmid DNA gene delivery to tumors. Int. J. Nanomedicine 8, 1573-1593. doi: 10.2147/IJN.S42800 
Deelman, L. E., Declèves, A. E., Rychak, J. J., Sharma, K., et al. (2010). Targeted renal therapies through microbubbles and ultrasound. Adv. Drug Deliv. Rev. 62, 1369-1377. doi: 10.1016/j.addr.2010.10.002

Deng, J., Huang, Q., Wang, F., Liu, Y., Wang, Z., Wang, Z., et al. (2012). The role of caveolin-1 in blood-brain barrier disruption induced by focused ultrasound combined with microbubbles. J. Mol. Neurosci. 46, 677-687. doi: 10.1007/s12031-011-9629-9

Dewitte, H., Vanderperren, K., Haers, H., Stock, E., Duchateau, L., Hesta, M., et al. (2015). Theranostic mRNA-loaded microbubbles in the lymphatics of dogs: implications for drug delivery. Theranostics 5, 97-109. doi: 10.7150/thno.10298

Hsu, P.-H., Wei, K. C., Huang, C. Y., Wen, C. J., Yen, T. C., Liu, C. L., et al. (2013). Noninvasive and targeted gene delivery into the brain using microbubble-facilitated focused ultrasound. PLoS ONE 8:e57682. doi: 10.1371/journal.pone.0057682

Huang, Q., Deng, J., Wang, F., Chen, S., Liu, Y., Wang, Z., et al. (2012). Targeted gene delivery to the mouse brain by MRI-guided focused ultrasound-induced blood-brain barrier disruption. Exp. Neurol. 233, 350-356. doi: 10.1016/j.expneurol.2011.10.027

Huang, S. L. (2008). Liposomes in ultrasonic drug and gene delivery. Adv. Drug Deliv. Rev. 60:1167. doi: 10.1016/j.addr.2008.03.003

Huang, Y., Alkins, R., Schwartz, M. L., and Hynynen, K. (2017). Opening the blood-brain barrier with MR Imaging-guided focused ultrasound: preclinical testing on a trans-human skull porcine model. Radiology 282, 123-130. doi: 10.1148/radiol.2016152154

Huang, Y., Ma, H., Wang, S., Shen, M., Guo, R., Cao, X., et al. (2012). Efficient catalytic reduction of hexavalent chromium using palladium nanoparticleimmobilized electrospun polymer nanofibers. ACS Appl. Mater. Interfaces 4:3054. doi: $10.1021 / \mathrm{am} 300417 \mathrm{~s}$

Hynynen, K., McDannold, N., Vykhodtseva, N., and Jolesz, F. A. (2001). Noninvasive MR imaging-guided focal opening of the blood-brain barrier in rabbits. Radiology 220, 640-646. doi: 10.1148/radiol.22020 01804

Kadkhodaei, B., Alvarsson, A., Schintu, N., Ramsköld, D., Volakakis, N., Joodmardi, E., et al. (2013). Transcription factor Nurr1 maintains fiber integrity and nuclear-encoded mitochondrial gene expression in dopamine neurons. Proc. Natl. Acad. Sci. U.S.A. 110, 2360-2365. doi: 10.1073/pnas.12210 77110

Kells, A. P., Forsayeth, J., and Bankiewicz, K. S. (2012). Glial-derived neurotrophic factor gene transfer for Parkinson's disease: anterograde distribution of AAV2 vectors in the primate brain. Neurobiol. Dis. 48, 228-235. doi: 10.1016/j.nbd.2011.10.004

Kim, T. W., Moon, Y., Kim,K., Lee, J. E., Koh, H. C., Rhyu, I. J., et al. (2011). Dissociation of progressive dopaminergic neuronal death and behavioral impairments by bax deletion in a mouse model of Parkinson's diseases. PLoS ONE 6:e25346. doi: 10.1371/journal.pone.0025346

Kirik, D., Georgievska, B., and Björklund, A. (2004). Localized striatal delivery of GDNF as a treatment for Parkinson disease. Nat. Neurosci. 7, 105-110. doi: $10.1038 / \mathrm{nn} 1175$

Kirik, D., Rosenblad, C., Bjorklund, A and Mandel, R. J. (2000). Long-term rAAVmediated gene transfer of GDNF in the rat Parkinson's model: intrastriatal but not intranigral transduction promotes functional regeneration in the lesioned nigrostriatal system. J. Neurosci. 20, 4686-4700.

Kumar, A., Kopra, J., Varendi, K., Porokuokka, L. L., Panhelainen, A., Kuure, S., et al., (2015). GDNF overexpression from the native locus reveals its role in the nigrostriatal dopaminergic system function. PLoS Genet. 11:e1005710. doi: 10.1371/journal.pgen.1005710

Kumari, A., and Yadav, S. K. (2011). Cellular interactions of therapeutically delivered nanoparticles. Expert Opin. Drug Deliv. 8, 141-151. doi: $10.1517 / 17425247.2011 .547934$

Lang, A. E., Gill, S., Patel, N. K., Lozano, A., Nutt, J. G., Penn, R., et al. (2006). Randomized controlled trial of intraputamenal glial cell line-derived neurotrophic factor infusion in Parkinson disease. Ann. Neurol. 59, 459-466. doi: 10.1002/ana.20737

Lei, Z., Jiang, Y., Li, T., Zhu, J., and Zeng, S. (2011). Signaling of glial cell line-derived neurotrophic factor and its receptor GFR $\alpha 1$ induce Nurr1 and Pitx3 to promote survival of grafted midbrain-derived neural stem cells in a rat model of Parkinson disease. J. Neuropathol. Exp. Neurol. 70, 736-747. doi: 10.1097/NEN.0b013e31822830e5
Lentacker, I., Geers, B., Demeester, J., De Smedt, S. C., and Sanders, N. N. (2010). Design and evaluation of doxorubicin-containing microbubbles for ultrasound-triggered doxorubicin delivery: cytotoxicity and mechanisms involved. Mol. Ther. 18:101. doi: 10.1038/mt.2009.160

Lin, C. Y., Hsieh, H. Y., Chen, C. M., Wu, S. R., Tsai, C. H., Huang, C. Y., et al. (2016). Non-invasive, neuron-specific gene therapy by focused ultrasoundinduced blood-brain barrier opening in Parkinson's disease mouse model. J. Control. Release 235, 72-81. doi: 10.1016/j.jconrel.2016.05.052

Lin, L. F., Doherty, D. H., Lile, J. D., Bektesh, S., Collins, F., et al. (1993). GDNF: a glial cell line-derived neurotrophic factor for midbrain dopaminergic neurons. Science 260, 1130-1132. doi: 10.1126/science.8493557

Liu, J., Chen, M., Wang, X., Wang, Y., Duan, C., Gao, G., et al. (2016), Piperine induces autophagy by enhancing protein phosphotase $2 \mathrm{~A}$ activity in a rotenone-induced Parkinson's disease model. Oncotarget 7, 60823-60843. doi: $10.18632 /$ oncotarget.11661

Liu, P., Wang, X., Zhou, S., Hua, X., Liu, Z., Gao, Y., et al. (2011). Effects of a novel ultrasound contrast agent with long persistence on right ventricular pressure: comparison with SonoVue. Ultrasonics 51, 210-214. doi: $10.1016 /$ j.ultras.2010.07.008

Matos, C., de Castro, B., Gameiro, P., Lima, J. L., and Reis, S. (2004). Zetapotential measurements as a tool to quantify the effect of charged drugs on the surface potential of egg phosphatidylcholine liposomes. Langmuir 20, 369-377. doi: $10.1021 / \mathrm{la} 034780 \mathrm{~b}$

Meng, F., Wang, J., Ding, F., Xie, Y., Zhang, Y., Zhu, J., et al. (2017). Neuroprotective effect of matrine on MPTP-induced Parkinson's disease and on Nrf2 expression. Oncol. Lett. 13, 296-300. doi: 10.3892/ol.2016.5383

Metz, G. A., and Whishaw, I. Q. (2002). Drug-induced rotation intensity in unilateral dopamine-depleted rats is not correlated with end point or qualitative measures of forelimb or hindlimb motor performance. Neuroscience 111, 325-336. doi: 10.1016/S0306-4522(02)00013-1

Meure, L. A., Foster, N. R., and Dehghani, F. (2008). Conventional and dense gas techniques for the production of liposomes: a review. AAPS PharmSciTech 9:798. doi: 10.1208/s12249-008-9097-x

Nitta, A., Nishioka, H., Fukumitsu, H., Furukawa, Y., Sugiura, H., Shen, L., et al. (2004). Hydrophobic dipeptide Leu-Ile protects against neuronal death by inducing brain-derived neurotrophic factor and glial cell linederived neurotrophic factor synthesis. J. Neurosci. Res. 78, 250-258. doi: $10.1002 /$ jnr.20258

Nosrat, C. A., Tomac, A., Lindqvist, E., Lindskog, S., Humpel, C., Strömberg, I., et al. (1996). Cellular expression of GDNF mRNA suggests multiple functions inside and outside the nervous system. Cell Tissue Res. 286, 191-207. doi: $10.1007 / \mathrm{s} 004410050688$

Nutt, J. G., Burchiel, K. J., Comella, C. L., Jankovic, J., Lang, A. E., Laws, E. R., et al. (2003). Randomized, double-blind trial of glial cell line-derived neurotrophic factor (GDNF) in PD. Neurology 60, 69-73. doi: 10.1212/WNL.60.1.69

Papademetriou, I. T., and Porter, T. (2015). Promising approaches to circumvent the blood-brain barrier: progress, pitfalls and clinical prospects in brain cancer. Ther. Deliv. 6, 989-1016. doi: 10.4155/tde.15.48

Patel, N. K., Pavese, N., Javed, S., Hotton, G. R., Brooks, D. J., Gill, S. S., et al. (2013). Benefits of putaminal GDNF infusion in Parkinson disease are maintained after GDNF cessation. Neurology 81, 1176-1178. doi: 10.1212/WNL.0b013e3182a55ea5

Paxinos, G., and Watson, C. (2007). The Rat Brain in Stereotaxic Coordinates, 6th Edn. Amsterdam: Academic Press/Elsevier.

Phillips, L. C., Klibanov, A. L., Wamhoff, B. R., Hossack, J. A., et al. (2010). Targeted gene transfection from microbubbles into vascular smooth muscle cells using focused, ultrasound-mediated delivery. Ultrasound Med. Biol. 36, 1470-1480. doi: 10.1016/j.ultrasmedbio.2010.06.010

Pranski, E. L., Dalal, N. V., Sanford, C. V., Herskowitz, J. H., Gearing, M., Lazo, C., et al. (2013). RING finger protein 11 (RNF11) modulates susceptibility to 6-OHDA-induced nigral degeneration and behavioral deficits through NF-кB signaling in dopaminergic cells. Neurobiol. Dis. 54:264. doi: $10.1016 /$ j.nbd.2012.12.018

Proft, J., Faraji, J., Robbins, J. C., Zucchi, F. C., Zhao, X., Metz, G. A., et al. (2011). Identification of bilateral changes in TID1 expression in the 6-OHDA rat model of Parkinson's disease. PLoS ONE 6:e26045. doi: 10.1371/journal.pone.0026045 Quintino, L., Manfré, G., Wettergren, E. E., Namislo, A., Isaksson, C., Lundberg, C., et al. (2013). Functional neuroprotection and efficient regulation of GDNF 
using destabilizing domains in a rodent model of Parkinson's disease. Mol. Ther. 21, 2169-2180. doi: 10.1038/mt.2013.169

Redmond, D. E., McEntire, C. R., Kingsbery, J. P., Leranth, C., Elsworth, J. D., Bjugstad, K. B., et al. (2013). Comparison of fetal mesencephalic grafts, AAV-delivered GDNF, and both combined in an MPTP-induced nonhuman primate Parkinson's model. Mol. Ther. 21, 2160-2168. doi: 10.1038/mt.201 3.180

Samiotaki, G., Acosta, C., Wang, S., and Konofagou, E. E. (2015). Enhanced delivery and bioactivity of the neurturin neurotrophic factor through focused ultrasound-mediated blood-brain barrier opening in vivo. J. Cereb. Blood Flow Metab. 35, 611-622. doi: 10.1038/jcbfm.2014.236

Sheffield, P., Trehan, A., Boyd, B., Wong, O. L., et al. (2008). Microbubbles as ultrasound contrast agents and in targeted drug delivery. Crit. Rev. Biomed. Eng. 36, 225-255. doi: 10.1615/CritRevBiomedEng.v36.i4.10

Tai, W., Ye, X., Bao, X., Zhao, B., Wang, X., Zhang, D. (2013). Inhibition of Src tyrosine kinase activity by squamosamide derivative FLZ attenuates neuroinflammation in both in vivo and in vitro Parkinson's disease models. Neuropharmacology 75, 201-212. doi: 10.1016/j.neuropharm.2013.07.020

Tenenbaum, L., and Humbert-Claude, M. (2017). Glial cell line-derived neurotrophic factor gene delivery in Parkinson's disease: a delicate balance between neuroprotection, trophic effects, and unwanted compensatory mechanisms. Front. Neuroanat. 11:29. doi: 10.3389/fnana.2017. 00029

Wang, F., Shi, Y., Lu, L., Liu, L., Cai, Y., Zheng, H., et al. (2012). Targeted delivery of GDNF through the blood-brain barrier by MRI-guided focused ultrasound. PLOS ONE 7:e52925. doi: 10.1371/journal.pone.0052925

Wang, X., Liu, P., Yang, W., Li, L., Li, P., Liu, Z., et al. (2014). Microbubbles coupled to methotrexate-loaded liposomes for ultrasound-mediated delivery of methotrexate across the blood-brain barrier. Int. J. Nanomedicine 9:4899. doi: $10.2147 /$ IJN.S69845

Weber-Adrian, D., Thévenot, E., O’Reilly, M. A., Oakden, W., Akens, M. K., Ellens, N., et al. (2015). Gene delivery to the spinal cord using MRI-guided focused ultrasound. Gene Ther. 22, 568-577. doi: 10.1038/gt.2015.25

Xu, A., Yao, M., Xu, G., Ying, J., Ma, W., Li, B., et al. (2012). A physical model for the size-dependent cellular uptake of nanoparticles modified with cationic surfactants. Int. J. Nanomedicine 7, 3547-3554. doi: 10.2147/IJN.S32188

Zhao, B., Chen, Y., Liu, J., Zhang, L., Wang, J., Yang, Y., et al. (2018). Blood-brain barrier disruption induced by diagnostic ultrasound combined with microbubbles in mice. Oncotarget 9, 4897-4914. doi: 10.18632/oncotarget.23527

Zhou, Y., Gu, H., Xu, Y., Li, F., Kuang, S., Wang, Z., et al. (2015). Targeted antiangiogenesis gene therapy using targeted cationic microbubbles conjugated with CD105 antibody compared with untargeted cationic and neutral microbubbles. Theranostics 5:399. doi: 10.7150/thno.10351

Conflict of Interest Statement: The authors declare that the research was conducted in the absence of any commercial or financial relationships that could be construed as a potential conflict of interest.

Copyright (๑) 2018 Yue, Miao, Gao, Zhao and Teng. This is an open-access article distributed under the terms of the Creative Commons Attribution License (CC $B Y)$. The use, distribution or reproduction in other forums is permitted, provided the original author(s) and the copyright owner are credited and that the original publication in this journal is cited, in accordance with accepted academic practice. No use, distribution or reproduction is permitted which does not comply with these terms. 